\title{
Dialectique de l'esthétique : Hans-Jürgen Krahl et le « marxisme occidental » entre art et politique
}

Andrea Cavazzini et Jérémy Hamers

\section{OpenEdition}

Journals

Édition électronique

URL : http://journals.openedition.org/grm/392

DOI : $10.4000 / \mathrm{grm} .392$

ISSN : $1775-3902$

Éditeur

Groupe de Recherches Matérialistes

Référence électronique

Andrea Cavazzini et Jérémy Hamers, « Dialectique de l'esthétique : Hans-Jürgen Krahl et le

« marxisme occidental » entre art et politique », Cahiers du GRM [En ligne], 5 | 2014, mis en ligne le 06

mai 2014, consulté le 13 juin 2020. URL : http://journals.openedition.org/grm/392 ; DOI : https://

doi.org/10.4000/grm.392

Ce document a été généré automatiquement le 13 juin 2020.

(c) GRM - Association 


\title{
Dialectique de l'esthétique : Hans- Jürgen Krahl et le « marxisme occidental » entre art et politique
}

\author{
Andrea Cavazzini et Jérémy Hamers
}

1 Hans-Jürgen $\mathrm{Krahl}^{1}$ (1943-1970) a été le théoricien le plus important du mouvement étudiant allemand des années 1960. Élève d'Adorno, il incarne une lecture de la Théorie critique de l'École de Francfort qui s'oppose radicalement à celle de J. Habermas et vise à réactiver le potentiel révolutionnaire du marxisme dialectique des années 1920-1930. Toutes ses interventions - recueillies dans Konstitution und Klassenkampf (Frankfurt a. M., Neue Kritik, 1971, 2008) - représentent des tentatives de construire une stratégie politique cohérente pour le SDS (Sozialistischen Deutschen Studentendbund). Krahl y impose souvent des glissements aux concepts de la philosophie classique allemande et $\mathrm{du}$ marxisme occidental afin d'en extraire des orientations politiques et organisationnelles ${ }^{2}$.

\section{Introduction}

par Andrea Cavazzini

2 Les notes fragmentaires que Hans-Jürgen Krahl a consacrées à l'esthétique témoignent du projet qui anime tous ses efforts théoriques ${ }^{3}$ : la politisation directe de la Théorie critique francfortoise et du «marxisme occidental» qui en est la matrice ${ }^{4}$. La confrontation avec la sphère des pratiques esthétiques, leur rapport à la philosophie et leur valeur politique, revêtent un rôle d'autant plus décisif dans ce projet que le marxisme occidental lui-même a décliné le diagnostic de la modernité capitaliste et sa critique dialectique dans les termes d'une critique de la culture dont la philosophie des arts est le moment central. La confrontation avec l'héritage culturel bourgeois et l'essor des avant-gardes et des expérimentations modernistes sont des moments décisifs de la problématique que Lukács, Benjamin, Adorno et Marcuse ont développée, et de leurs analyses du capitalisme moderne et des tentatives révolutionnaires au XXe siècle. Les 
réflexions de Krahl s'inscrivent dans cette problématique que semblent réactiver les perspectives d'une "révolution en Occident » dans les pays capitalistes avancés entre les années 1950 et 1960. Mais les théoriciens du mouvement étudiant, dont Krahl fait partie, considèrent cette critique du capitalisme rationalisé et de la réification comme abstraite et spéculative : le problème se pose dès lors d'une réalisation de la Théorie critique par une destruction pratique des conditions de l'hétéronomie et de la domination dans le capitalisme avancé. C'est dans ce contexte que la réflexion esthétique des marxistes occidentaux fait l'objet de tentatives visant à dépasser son statut purement contemplatif de critique de la culture ${ }^{5}$.

3 La position que Krahl élabore dans ses notes consiste à ramener le problème de la réalisation de la critique esthétique à la problématique spéculative du dépassement de l'idéalisme qui a dominé la philosophie post-hégélienne. Krahl ne propose en effet aucune stratégie concrète pour l'action politique dans la sphère culturelle: ses réflexions visent uniquement à situer l'expérience artistique des avant-gardes et du modernisme dans la crise de la fausse conciliation idéaliste. Pour lui, la sphère esthétique post-classique exprime un refus de la conciliation et une critique de la réification qui restent impolitiques. Au contraire, la praxis révolutionnaire invoquée par Marx vise à dépasser pratiquement les scissions entre individu et genre, sensible et intelligible, singulier et universel, que l'idéalisme ne pouvait réconcilier que dans l'Idée - le Beau étant l'un des moments de cette réconciliation abstraite qui ne peut que reproduire la réification. La critique révolutionnaire de l'idéalisme reconnaît ainsi la légitimité, mais aussi les limites, de la critique esthétique de la société capitaliste dans ses différentes figures (décadentisme, avant-gardes). Elle la reconnaît comme une protestation contre la réification, mais qui reproduit la logique de la réification, qui en utilise les expressions subjectives sans parvenir à les dépasser. C'est pourquoi Krahl insiste sur les déchirures subjectives du caractère décadent considéré comme le corrélat subjectif de la position critique limitée à la sphère esthétique : la vie mutilée se limite à se lever contre ses propres mutilations sans pouvoir changer de terrain.

4 La généalogie que Krahl esquisse s'inscrit entièrement dans la vexata quaestio des rapports entre le marxisme et la culture classique allemande (que résument les noms de Hegel, Goethe, Schiller, Kant, Humboldt...) : la praxis révolutionnaire appelée de ses vœux par le jeune Marx est considérée comme une "solution» des apories de la philosophie spéculative et de l'humanisme esthétique. L'idée de cette continuité avait inspiré, non seulement la problématique générale du « marxisme occidental » et de la Théorie critique, mais aussi la politique culturelle que György Lukács avait proposée dans les années 1930. Le concept de "décadence " est à cet égard décisif dans la reconstruction lukácsienne du devenir de la culture bourgeoise : il indique à la fois la réaction idéologique du monde bourgeois contre le mouvement prolétarien et la partie de la culture bourgeoise qui refuse d'être " héritée " par le communisme. Autrement dit, la culture bourgeoise se livre à la décadence dans la mesure où elle renonce, face à l'essor de la lutte de classes du prolétariat, à ses idéaux d'émancipations, que le marxisme devra et pourra relayer, en les incorporant à la perspective communiste tout en les libérant de leurs contradictions. Dans le schéma lukácsien, l'horizon communiste hérite de la grande culture bourgeoise, et la culture bourgeoise trouve dans le communisme sa vérité. La décadence représente la rupture de ce rapport et de cette transmission. Elle est caractérisée par un schéma historique et théorique que Krahl relaie dans ses notes : 
Une des questions critiques centrales de la préparation idéologique de l'Allemagne à la Révolution de 1848 est le débat autour de la dissolution de l'hégélianisme. Ce processus de dissolution caractérise la fin de la dernière grande philosophie de la société bourgeoise. Simultanément ce processus devient un élément important de la genèse du matérialisme dialectique (...). [La] décadence apparaît avec la prise du pouvoir politique par la bourgeoisie et le déplacement en position centrale de la lutte des classes entre bourgeoisie et prolétariat ${ }^{6}$.

5 Le titre de l'essai lukácsien utilise le terme Verfall (« déclin »), pas celui de Dekadenz. Chez Marx, ce mot désigne l'incapacité de la pensée économique post-classique à reconnaître les contradictions de la société capitaliste - la nécessité structurelle des crises et de l'exploitation - que Ricardo et Smith avaient été capables de formuler tout en restant dans l'horizon bourgeois. Pour Marx, le " déclin » est un déclin théorique, qui coïncide avec une volonté apologétique de construire dans l'idéologie des harmonies miraculeuses et des conciliations verbales au lieu de reconnaître le réel irréductible des conflits sociaux : les cibles de cette critique sont des théoriciens comme Malthus, James et John Stuart Mill, Frédéric Bastiat, etc. Aussi lorsque Lukacs emploie le mot Dekadenz comme synonyme de Verfall en vient-il à transformer le concept marxien en diagnostic général portant sur la crise de la civilisation. D'abord, parce que, dans la mesure où le mot Dekadenz renvoie au Décadentisme, le domaine esthétique devient le domaine privilégié où l'analyse de la crise culturelle bourgeoise pourra être menée. Ensuite parce que l'introduction de la notion de Dekadenz permet à Lukács d'articuler la phénoménologie des positions subjectives associées au devenir des pratiques esthétiques depuis la fin du XIXe siècle aux stratégies que la bourgeoise déploie pour éviter toute confrontation traumatique avec le réel historico-social: l'esthétisme, le sentiment d'anomie, la fascination pour la mort et le macabre, la perte de réalité, la précarité des repères objectifs et subjectifs - tous ces traits subjectifs du « caractère décadent ", qui correspondent à des thèmes ou à des styles dans la sphère des arts, deviennent pour Lukács des «apologies indirectes» de la réalité capitaliste, par lesquelles la bourgeoisie décadente renonce à assumer une position active et créatrice devant l'histoire.

Par cette opération, Lukács fait d'une notion marxienne relativement anodine le pivot d'une analyse marxiste-occidentale de la crise de la civilisation bourgeoise. La problématique qui domine cette analyse s'inspire des critiques de la civilisation européenne moderne menées par Max Weber et par Georg Simmel, et dont le précurseur est incontestablement Nietzsche. C'est ce dernier, d'ailleurs, qui a élevé le mot Dekadenz au rang de concept de la philosophie de l'histoire et de la critique de la civilisation : chez l'auteur de Zarathoustra, ce concept a un statut très proche de celui que lui assignera Lukács - la décadence est l'incapacité à instaurer un rapport authentique et créateur à la réalité historique, qui se traduit par une subjectivité fragmentée, instable et incohérente, incapable de se référer à une Totalité du sens et de la valeur. Dekadenz signifie ainsi avant tout la dissolution de cette Totalität que la pensée classique allemande avait érigée en horizon idéal sous les formes de la construction spéculative de l'Absolu et de l'humanisme esthétique. Le « déclin » de la bourgeoisie se manifeste dans l'incapacité, non seulement de tolérer les contradictions, mais aussi de se confronter à une réalité contradictoire sans céder sur l'horizon d'émancipation que promet l'idéal bourgeois de l'homme harmonieux. Les grands théoriciens de l'« aspiration à l'harmonie entre les aptitudes et les forces de l'homme ", " depuis la Renaissance jusqu'à Hegel en passant par Winckelmann $»^{7}$, ont reconnu et formulé la 
contradiction entre le déploiement maximal de toutes les puissances collectives de l'humanité - ce qui implique le développement du commerce, de la technologie et de l'État moderne - et les effets néfastes que ce déploiement exerce sur les êtres humains, dans la mesure où ces développements ne peuvent avoir lieu que dans une société divisée en classes :

Les grands hommes de la Renaissance ont travaillé au développement de toutes les forces productives de la société. Leur grandiose objectif était (...) la création d'un état social où seraient libérées toutes les capacités humaines, toutes les possibilités de connaître à fond les forces de la nature et de les soumettre à fond aux buts de l'humanité (...). [Mais] plus les forces productives du capitalisme se développent, plus vigoureusement se déploie l'effet asservissant de la division capitaliste du travail. La période de la manufacture fait déjà de l'ouvrier un spécialiste étroit, ossifié, d'une seule opération, et l'appareil d'État de cette époque commence déjà à transformer ses employés en bureaucrates sans idées ni âme (...). [Chez les penseurs importants des Lumières] l'âpre critique de la division capitaliste du travail cohabite toujours, immédiatement mais sans lien avec elle, avec l'encouragement énergique au développement des forces productives (...). Les grands poètes et esthéticiens des Lumières et de la première moitié du XIXe siècle (...) ne sont pas non plus en mesure de résoudre les contradictions existantes de la société capitaliste. Leur grandeur et leur audace tiennent à ce que (...) ils critiquent impitoyablement la société bourgeoise et cependant ne renoncent jamais un seul instant à s'affirmer pour le progrès 8 .

C'est à partir des apories des Lumières face à la contradiction immanente à la société bourgeoise-capitaliste que Lukács déduit la position des "classiques » allemands. La théorie esthétique du classicisme allemand produit une critique de la division du travail tout en assignant à l'art la fonction d'un dépassement non politique des contradictions: «Les poètes et penseurs du classicisme allemand, dont l'activité décisive coïncide déjà avec la période qui a suivi la Révolution française, cherchent diverses solutions utopiques. Leur critique de la division capitaliste du travail n'en est pas moins aiguë que celle des hommes des Lumières. Eux aussi soulignent avec une rigueur croissante le morcellement de l'homme (...). [Ils] cherchent l'harmonie de l'homme et la beauté correspondante dans l'art (...). L'activité esthétique prend de ce fait chez eux une signification éminente et souvent exagérée d'idéalisme. Ils ne voient pas seulement dans l'harmonie artistique un reflet et une expression de l'homme harmonieux, mais le principal moyen pour surmonter intérieurement le déchirement et la déformation de l'homme provoqués par la division capitaliste du travail. Cette problématique entraîne nécessairement un renoncement à accomplir le dépassement du caractère inharmonieux de la vie capitaliste dans celle-ci, telle qu'elle est (...). Goethe et Schiller croient que de petits groupes d'hommes sont en mesure de réaliser pratiquement l'idéal de la personnalité harmonieuse et peuvent ainsi constituer des cellules qui répandront cet idéal dans l'humanité tout entière (...). Une telle croyance constitue aussi le fondement de Wilhelm Meister; c'est sur de telles espérances utopiques que s'achève Sur l'éducation esthétique de l'homme, l'œuvre de Schiller "9. Mais la réconciliation esthétique que recherchent Goethe et Schiller devient impossible déjà dans les premières décennies du XIXe siècle :

Hegel et Balzac vivent déjà les contradictions de la division capitaliste du travail sous une forme beaucoup plus développée que Goethe et Schiller au temps de leur collaboration (...). [Ils] voient avec une acuité impitoyable le caractère inhumain de la société capitaliste, ils la voient écraser toute harmonie humaine chez tout homme et dans toutes les manifestations de la vie des hommes (...). Balzac montre précisément comment la société capitaliste engendre avec la nécessité d'une loi 
d'airain la dissonance et la laideur dans toutes les manifestations de la vie, de quelle manière sont foulées aux pieds sans pitié toutes les aspirations à une vie belle et harmonieuse ${ }^{10}$.

8 Le roman du XIXe siècle représente par conséquent, dans la sphère des formes artistiques, l'impossibilité de réconcilier ces dissonances à travers les effets bénéfiques de la « belle apparence » (Schein) théorisée par Schiller :

Les grands réalistes du capitalisme déjà épanoui (...) sont forcés, du fait qu'ils figurent fidèlement la réalité, de renoncer délibérément à la représentation de la vie belle, de l'homme harmonieux. S'ils veulent être de grands réalistes, ils ne peuvent figurer que la vie inharmonieuse, déchirée, la vie qui écrase sans pitié et même, ce qui est pire, déforme intérieurement, corrompt et traîne dans la boue tout ce qu'il y a de grand et de beau en l'homme. Le résultat final auquel ils aboutissent est forcément que la société capitaliste est un vaste cimetière où reposent la grandeur et l'authenticité humaine assassinées ${ }^{11}$.

9 C'est à partir de cette représentation «impitoyable » de la déformation et de la corruption que s'affirme la position proprement décadente. La subjectivité décadente fonde ses propres formes d'expression artistique à partir des déchirures subjectives imposées par la vie en régime capitaliste. Elle représente une protestation véhémente contre ces déchirures, mais elle n'arrive jamais à les saisir autrement que comme un destin indépassable coöncidant avec l'essence ultime de l'existence de l'homme :

À l'intérieur de cette dénonciation accusatrice de la société capitaliste, les chemins se séparent (...). Le résultat produit par le capitalisme, c'est-à-dire la destruction de l'homme, devient-il sans plus attendre le fondement de la figuration artistique - ou bien la lutte contre cela est-elle également figurée, ainsi que le sublime et la beauté des forces humaines qui subissent la destruction et qui se révoltent contre elle avec plus ou moins de succès ? ${ }^{12}$

10 La figuration esthétique des forces qui s'opposent à la mutilation est une tâche artistique, qui implique que l'artiste rattache son travail à la tradition que Lukács vient d'évoquer - la tradition bourgeoise classique, des Lumières au roman français et russe en passant par le classicisme allemand. Mais cette tâche est également éthique. L'artiste authentique ne doit pas désespérer de la possibilité de s'opposer au capitalisme: sa capacité à représenter les traces de l'harmonie humaine suppose qu'il reconnaisse dans l'actualité l'existence réelle des tendances historiques qui témoignent de la possibilité concrète de cette harmonie :

[Il y a toute une série d'écrivains] qui figurent avec l'intensité de la vie la lutte de chaque jour, voire de chaque heure, que l'homme de ce temps doit mener contre l'environnement capitaliste afin de conserver son intégrité humaine. Il est condamné à succomber dans cette lutte s'il s'en remet à ses seules forces individuelles; il ne peut la mener que s'il trouve un lien vivant avec les forces populaires qui garantissent la victoire finale de l'humanisme au plan économique et politique, social et culturel ${ }^{13}$.

11 Les écrivains qui incarnent cette position sont Anatole France, Sinclair Lewis, Thomas Mann, Heinrich Mann ou Maxime Gorki. Par contre, d'autres écrivains, souvent plus radicaux du point de vue des positions politiques explicites, "s'efforcent de représenter non pas la lutte contre la destruction mais le monde détruit; non pas le processus vivant mais le résultat mort (...) des hommes et des destins qui contenaient les traits les plus frappants de la destruction sociale de l'homme par le capitalisme, alignés comme dans une chronique $\aleph^{14}$. La révolte radicale se paie dans ce cas d'une capitulation vis-à-vis de la pure surface de la réalité capitaliste et débouche sur une représentation mécanique et inarticulée de la vie mutilée : la dissolution des formes 
esthétiques témoigne ainsi, chez les artistes d'avant-garde, de leur incapacité à reconnaître les forces qui s'opposent à la dissolution de l'homme.

La stratégie culturelle de Lukács précède l'adoption par le Komintern de la ligne des fronts populaires antifascistes et de l'unité entre les forces révolutionnaires et les courants démocrates - une ligne que Lukács avait prônée avant son imposition par Staline en 1934-1935. Pourtant, la visée lukácsienne ne saurait être réduite à l'adhésion du philosophe à une ligne politique contingente. En réalité, ce que visait Lukács était beaucoup plus ambitieux. Il s'agissait, d'une part, de "civiliser» le communisme soviétique en lui léguant les traditions culturelles de la bourgeoisie dans sa phase révolutionnaire ${ }^{15}$; et, d'autre part, de proposer à la conscience malheureuse des intellectuels bourgeois une perspective anti-capitaliste susceptible de canaliser leur opposition morale et esthétique à la société moderne :

[La division du travail physique et intellectuel] différencie le travail intellectuel en divers domaines séparés, qui se mettent à représenter des intérêts particuliers, matériels et intellectuels, se faisant concurrence, et forment par conséquent une espèce particulière de spécialistes (...). Le trait particulier du développement capitaliste (...) consiste en ce que les classes dominantes y sont également soumises à la division du travail. Tandis que les formes plus primitives de l'exploitation, en particulier celles de l'économie esclavagiste gréco-romaine, ont créé une classe dominante qui est demeurée pour l'essentiel épargnée par la division du travail, cette dernière, dans le capitalisme (...), s'étend aussi aux membres de la classe dominante dont la "spécialité" consiste à ne rien faire. Par conséquent, la division capitaliste du travail ne se soumet pas seulement l'ensemble des domaines de l'activité matérielle et intellectuelle, mais s'enfonce profondément dans l'âme de chaque homme et y cause de profondes déformations qui apparaissent ensuite, sous des formes diverses, dans les divers modes d'expression idéologiques. Quand la pensée et la création littéraire décadentes se soumettent sans lutte à ces effets de la division du travail, quand elles acceptent sans contradiction les déformations psychologiques et morales qui en découlent, quand elles vont jusqu'à les approfondir encore et à les parer de vertus, on a là un des traits essentiels les plus importants de la période de décadence ${ }^{16}$.

13 Les scissions subjectives produites par la structure sociale capitaliste concernent la bourgeoisie et déterminent la possibilité qu'une conscience oppositionnelle se développe chez ses représentants intellectuels :
La bourgeoisie ne possède que l'apparence d'une existence humaine. C'est pourquoi, chez tout individu issu de la classe bourgeoise, doit surgir une contradiction vivante entre l'apparence et la réalité et il dépend alors largement de l'individu lui-même qu'il laisse endormir cette contradiction sous l'effet des anesthésiants idéologique que sa classe lui impose sans cesse ou alors que la contradiction reste vivante en lui- même (...). Il n'est pas du tout inévitable que dès l'origine sa rébellion contre cette apparence contienne une tendance (...) à la rupture avec sa propre classe. Dans la vie même, de telles rébellions partielles des individus se produisent sans cesse et massivement, mais on doit ajouter qu'en particulier dans les conditions de la décadence générale, il faut que l'individu ait une grande force intellectuelle et morale pour trouver ici une issue ${ }^{17}$.

Lukács assigne aux intellectuels bourgeois la tâche de ne pas désespérer, de persévérer dans leur confiance en la possibilité pour l'homme de réaliser l'harmonie de ses facultés même au milieu des ravages induits par la réification. La « cage d'acier » du système capitaliste impose des formes de subjectivité dont la mutilation atteint le paroxysme à l'époque de l'impérialisme: 
La société apparaît comme une puissance incompréhensible, mythique, dont l'objectivité fataliste, privée de toute humanité, se dresse face à l'individu, menaçante et incomprise (...). Cette évacuation de l'activité sociale a pour l'homme isolé la conséquence idéologique nécessaire que désormais sa vie privée se déroule apparemment - en dehors de cette société mythifiée (...). Le "petit homme" rampant et arriviste de la vie professionnelle déchaîne dans son propre foyer tous ses instincts de puissance comprimés et pervertis. Mais on ne peut, par le biais d'un reflet déformé (...) supprimer le rapport objectif des phénomènes sociaux. Même dans le cercle étroit, idéologiquement circonscrit, de la vie privée, le caractère social fait valoir ses droits. L'amour, le mariage, la famille sont des catégories sociales objectives, des "formes ontologiques", des "déterminations existentielles" de la vie humaine ${ }^{18}$.

Dans le monde réifié la vie privée semble s'opposer radicalement à la sphère historicopolitique. La sphère personnelle et la vie intime apparaissent comme les lieux de l'authenticité anhistorique et asociale de l'âme. Cet isolement mythologisé de la vie intime et intérieure est lui-même un symptôme de la réification. En réalité, les mouvements intimes de l'âme gardent toujours un lien avec des modes élémentaires de l'histoire collective. Les structures de la vie réifiée sont toujours des structures sociales, par lesquelles chaque être humain est relié à ses semblables. Dans ces relations objectives et irréductibles réside la possibilité de développer une position subjective qui viserait la totalité des relations humaines, par-delà la réification et le morcellement. C'est à partir de cette intention vers la totalité qui persiste jusque dans les traces les plus infimes et imperceptibles de la résistance aux effets du capitalisme que les formes esthétiques doivent déployer leurs figurations :

La figuration peut bien montrer que les hommes vivant aujourd'hui sont de misérables fragments et caricatures de l'homme; l'écrivain doit avoir éprouvé en lui-même les possibilités de la véritable condition humaine, de son achèvement harmonieux et de sa richesse, afin de pouvoir voir les caricatures comme caricatures et, à partir de la pulvérisation des hommes en fragments, d'éprouver et d'éveiller une disposition à la lutte contre le monde qui produit cela quotidiennement ${ }^{19}$.

16 La position de Lukács pourrait être caractérisée comme une reprise marxiste de l'éducation esthétique théorisée par Schiller. C'est par opposition à ce projet que les théories de l'École de Francfort vont élaborer une version différente de la dialectique de l'esthétique. À l'humanisme esthétique schillérien que Lukács propose de réactiver, Adorno et Horkheimer opposeront, dans la Dialektik der Aufklärung, une vision des arts et de leur fonction s'inspirant des grandes expérimentations modernistes et dont le principe formel décisif est l'accentuation impavide de la dissonance :

Les grands artistes n'ont jamais été ceux qui incarnaient le style le plus pur et le plus parfait, mais ceux qui, dans leurs œuvres, utilisèrent le style pour se durcir eux-mêmes contre l'expression chaotique de la souffrance comme vérité négative. Le moment qui, dans l'œuvre d'art, lui permet de transcender la réalité, est en effet inséparable du style; il ne consiste cependant pas en la réalisation d'une harmonie, d'une unité problématique entre la forme et le contenu, entre l'extérieur et l'intérieur, entre l'individu et la société, mais dans les traits où affleure la contradiction, dans l'échec nécessaire de l'effort passionné vers l'identité. Au lieu de s'exposer à cet échec dans lequel le style de la grande œuvre d'art s'est toujours nié, l'œuvre médiocre s'en est toujours tenue à sa similitude avec d'autres, à un succédané d'identité ${ }^{20}$.

17 Cette position ne fait que renverser celle de Lukács : l'art est toujours l'organon d'une totalité que le capitalisme a éclipsée de la vie des sociétés contemporaines. Mais pour le 
philosophe hongrois le grandes œuvres esthétiques doivent déployer ces germes de totalité dont témoigne l'aspiration persistante des hommes à la santé et à l'authenticité, tandis que pour Adorno et Horkheimer la totalité ne peut être cernée que négativement, à travers l'approfondissement des déchirures dans lesquelles se manifeste la révolte du particulier non réconcilié contre la fausseté de toute totalisation donnée. Finalement, ces deux positions convergent sur un point: elles assignent à la sphère esthétique la fonction d'incarner une totalité du sens impossible à retrouver dans l'action historico-politique. Les arts deviennent l'ersatz d'une transformation réelle des rapports sociaux que les impasses du mouvement communiste, la dérive stalinienne de la Révolution russe, et la transformation de la société bourgeoise en "cage d'acier» capitaliste rendent impraticable. Mais tant la position de Lukács que celle des francfortois deviennent vite intenables: «Parler de Goethe au soldat de l'Armée Rouge "aux mains sanglantes et vides" auquel Brecht avait consacré un de ses poèmes de jeunesse : tel fut l'honneur de Lukács. Son erreur : croire que cette vision aurait pu survivre à la période ouverte par l'assassinat de Kirov (décembre 1934) et dont la conclusion fut le pacte germano-soviétique (août 1939) »" Quant à l'artiste dont la fonction oppositionnelle coïnciderait avec l'expérimentation incessante des modes de figuration du négatif et de la scission, cette fonction tend à devenir indiscernable de la marginalité de l'esthète, voire du conformisme du «fou du roi », dans le contexte de la stabilisation interne des sociétés capitalistes.

18 Autrement dit, ni Lukács ni Adorno et Horkheimer ne surent proposer une solution valable du problème de l'inscription sociale et politique des pratiques esthétiques - et des pratiques intellectuelles en général - dans la séquence déterminée par les conséquences de la Révolution d'octobre et de la guerre mondiale. Lorsque ce problème se représente entre l'après-guerre et le début des années 1960, la conjoncture est profondément différente. D'abord, les figures d'intellectuels qui étaient les destinataires des stratégies de Lukács et d'Adorno-Horkheimer ont commencé à devenir anachroniques. L'humaniste-homme des Lumières, l'esthète romantique ou décadent et le Génie moderniste tendent à disparaître en tant que figures sociales à l'époque du capitalisme avancé technocratique, de l'industrie culturelle et de la scolarisation de masse. Les nouvelles formes de l'intellectualité sont précisément celles qui déterminent la pratique et la réflexion des mouvements étudiants en Europe occidentale : l'intellectuel-masse fait son apparition, déterminé par les transformations de l'école et de l'université, des moyens de communication ou de l'édition, et son destin politique devient un enjeu impossible à traiter suivant les référentiels des années 1920-1930. La reformulation théorique de la dialectique de l'esthétique reste, chez Krahl, politiquement muette, tandis que la stratégie politique que le théoricien du SDS élabore dans les années 1960 s'adresse à l'intellectualité techno-scientifique, et se fonde explicitement sur l'obsolescence assumée des formes bourgeoises d'existence du savoir et de la culture.

19 Dans les «séquences rouges " européennes de la deuxième moitié du XXe siècle, les mouvements étudiants et les nouvelles gauches thématisent sous des formes différentes l'essor de figures sociales de l'intellectualité que les anciens référentiels politiques et théoriques étaient incapables de traiter. Après la fin de ces séquences, aucune proposition d'action commune et d'organisation n'a été adressée à ces figures dont la présence est devenue extrêmement massive et multiforme, nourrissant une large partie des vastes armées de la précarité, mais aussi de ce qui reste des institutions 
de la culture, de la recherche, de l'enseignement, et de l'intervention sociale. Il faudrait ouvrir une longue discussion, que nous ne pouvons qu'évoquer ici. Nous conclurons ces remarques sur les notes de Krahl par un autre point. La dialectique de l'esthétique développée par la pensée classique allemande et léguée au marxisme occidental se fondait sur un décalage nécessaire entre les raisons et les formes de l'art et celles de la politique. L'intention dirigée vers la totalité qui se manifeste dans les objectivations de l'art - en réalité dans toute objectivation de la culture - doit sa spécificité et ses pouvoirs à des strates de l'expérience humaine dont la temporalité est irréductible à celle de l'action politique: Lukács l'avait bien vu en parlant de «déterminations ontologiques » qui structurent tant la lutte contre la vie mutilée que l'expression de cette lutte dans les formes artistiques. Or, c'est déjà sur cette idée que se fondaient l'humanisme esthétique de Schiller et la pédagogie historico-philosophique de Lessing: en l'absence de solution politique effective, les objets de la culture peuvent, grâce à leur enracinement dans une temporalité plus longue et plus dense, assurer la continuité d'un discours qui, sans être celui de la politique, touche aux raisons ultimes de la politique $^{22}$. L'un des problèmes majeurs de l'époque présente est le statut de ce discours, la possibilité de sa survie par-delà l'effondrement des projets politiques qui s'en étaient nourris. Cet effondrement a contribué puissamment à dissoudre les formes et les repères traditionnels de l'agir culturel. Les formes institutionnelles par lesquelles la société bourgeoise européenne avait territorialisé et défini la sphère de la culture ont perdu leur consistance et leur autonomie; l'industrie culturelle contemporaine ne reconnaît plus aucune sphère de l'« esprit " séparée de la "vie ». Les analyses que Hans-Magnus Enzensberger consacre à la littérature sont valables pour n'importe quel domaine au sein de ce qui s'appelait autrefois la culture :

De temps immémorial, l'interpénétration de notre travail avec le travail d'autres hommes, notre dépendance mutuelle, n'ont cessé de croître (...). En ce qui concerne la socialisation [capitaliste] du travail - un processus dont la fin n'est pas encore en vue -, le Dr Marx ne s'est pas trompé. L'institution littéraire n'est que l'une d'entre ses plus modestes victimes. Le privilège dont elle jouissait jadis, sa compétence particulière, se sont dissous comme un cachet d'Alka-Seltzer. La poésie s'étale maintenant partout, dans les manchettes des journaux, la musique pop, la publicité, et que sa qualité laisse à désirer ne fait rien à l'affaire. Le cinéma, toutes sortes de thérapies, la mode, la musique, l'action politique, les sectes et les cultures underground, le spectacle extravagant qu'offrent les rues de nos métropoles, inventent des sentiments inconnus jusqu'ici, de nouvelles formes de perception ${ }^{23}$.

Inutile d'opposer à cette situation la nostalgie de l'époque "classique " de la Haute Culture : l'intellectuel-pédagogue, l'écrivain humaniste et l'esthète décadent sont à jamais des figures du passé, et le statut "gazeux», diffus et acéphale de l'activité culturelle contemporaine correspond à la multiplication des intellectuels-masses en tant que figures de la subalternité au sein d'une industrie culturelle qui tend à s'incorporer aux sphères de la production et de la consommation. C'est à partir de ces figures que des propositions concernant l'agir et les objets culturels devraient être élaborées. Mais ces propositions ne devraient pas négliger la recherche, dans les formes contemporaines de la culture, de ce discours sur les fins et les principes qui, « de temps immémorial », habite les œuvres de l'esprit :

Regardez ce verre d'eau, ne quittez pas des yeux le cachet blanc et dur d'AlkaSeltzer que j'y plonge et observez ce qui se passe... L'institution se dissout, mais ne disparaît pas. Elle est toujours là, mais on ne la remarque plus. Dissoute, éparse et finement répartie, elle continue d'exister, ayant gagné en ubiquité ce qu'elle a perdu en concentration. Et ne négligez pas cet autre détail, qui pourrait se révéler 
d'une grande importance, bien que rien ne nous permette d'en être sûrs : le dépôt. Si vous y regardez de près, vous remarquerez en effet un dépôt blanchâtre au fond $\mathrm{du}$ verre - le tenace résidu du concentré originel, qui a manifestement résisté à la dissolution. Ce simple reste, qui paraît ne jouer aucun rôle, comparé au courant principal, vous pouvez bien sûr l'ignorer, mais qui sait ?... ${ }^{24}$

\section{Notes de Hans-Jürgen Krahl}

\section{Sur le rapport entre esthétique et métaphysique (et sur Nietzsche)}

Lêtre vrai [das wahre Sein] et en les frappant avec les occasions répétées de l'immanence provoquées par le malheur. Le caractère démythologisant de l'art. Par ailleurs, le beau possède aussi un index, obstinément ontologique et réifiant [verdinglichenden], du dédoublement réfléchissant de ce qui est (essence tragique de la métaphysique et essence métaphysique du tragique). L'idéologie dans l'art réside dans le fait que l'essence en tant que beau simule sa présence, feint son apparition : le caractère réifié.

\section{Esthétique}

L'esthétique matérialiste comme philosophie de l'histoire de l'art ${ }^{26}$, là où l'œuvre d'art se résout totalement dans sa temporalité et dans son caractère historique d'œuvre d'art: À la recherche du temps perdu (Proust). Remémoration, l'expérience faite après 
coup, post festum, indissociable de la compréhension, c'est le médium de l'art. Chez Beckett, les hommes ont perdu leurs mémoires, leur souvenir ${ }^{27}$ (Kafka : l'animal avec un passé, remémoration/métamorphose) (1). L'art destiné à l'imitation [Nachahmung] de la nature bannit l'esprit dans ce qui lui est étranger, mais, en même temps, dépasse cela souverainement.

Le développement d'instruments de production vainc la nature par la ruse à travers une imitation conséquente. Hegel rompt avec cela. L'art comme lieu de la remémoration s'affranchit de la domination de la nature, noyau temporel de l'art: remémoration? (Euménide de l'esprit séparé) Entendre l'herbe qui pousse, écouter la naissance [Entstehung] des feuilles (Hoffmannsthal), commencer à découvrir le secret de la genèse : cela fait partie de l'esprit métaphysique de l'art ; il simulait cela, le traitait de façon réflexive dans l'esthétisme, qui relève donc éminemment de la philosophie de l'histoire.

\section{Décadence}

Il est caractéristique des théories philosophiques de la bourgeoisie tardive que le concept ne peut et ne veut plus trouver la force de la cohérence du système qui englobe tout et qui ne laisse tout simplement rien de côté. Le concept devient fragile, il fuit dans le clair-obscur métaphorique et allégorique [allegoriale] du paraître [Schein] esthétique [illisible]. Mais les fissures du concept sont précisément ces differentia specifica, dans lesquelles une lueur de cet individuel [jenes Individuellen] surgit, précisément cet individuel que la philosophie systématique des siècles récents et passés s'est efforcée d'articuler en vain.

Mais la vie, qui se représente esthétiquement dans le concept se sachant impuissant, est aussi fissurée et fragile que le concept ; la vie ne vit pas (2). Le travail philosophique de Nietzsche montre non seulement que la vie de l'esprit - cette vie que la grande philosophie idéaliste s'illusionnait d'avoir reconnue comme la substance de la réalité était un semblant [Schein] trompeur, mais aussi que la vie immédiate elle-même devint [illisible] une intelligibilité morte, une réflexion malheureuse. La vie est semblant [Schein] - c'est la dernière certitude de Nietzsche; ce semblant est ivresse et destruction, l'esprit décadent de la musique, le semblant malheureux qui se présente comme vie, peut, dans le meilleur des cas, être une euphorie momentanée, l'étroit éclair d'or sur le ventre du serpent vita (3).

La critique contre le concept pur de l'abstraction idéaliste débouchait chez Marx sur une critique de la philosophie qui exigeait que sa substance [Inbegriff], la raison [Vernunft], soit réalisée socialement par l'action révolutionnaire d'un sujet historique. On ne parvient à entrer dans un médium concret qu'en réalisant les idées régulatrices de la raison philosophique, qu'en relevant [aufheben] le mundus intelligibilis dans le mundus sensibilis, qu'en formant l'être humain, à travers l'émancipation de la sensibilité, en être humain générique [Gattungswesen (4)] - la médiation entre le général et le particulier avec et après Kant (héritage des Lumières) fut assimilée par l'intérêt rationnel et émancipateur de la philosophie de l'histoire. Cette médiation s'articula d'abord [illisible] par la médiation entre théorie et praxis, restée obscure chez Kant, dans le médium des théories esthétiques de la philosophie de l'art.

Le philosophe des âges du monde Schelling (5) l'élève à [illisible] la dignité métaphysique, et le disciple de Kant, Schiller (6) (à la fois néoclassique et homme des 
Lumières), la relie à l'intérêt pédagogique de l'éducation du genre humain [Erziehung des Menschengeschlechts (7)] dans une perspective historico-universelle [universalhistorischer Absicht] et l'articule dans le médium de la théorie esthétique.

31 Tout comme la critique marxiste du concept idéaliste, la critique bourgeoise cherche également après Hegel à atteindre un médium concret. Mais alors que Marx proclame la relève [Aufhebung] révolutionnaire de la raison philosophique, dont la réalisation devient manifeste par la critique matérialiste de l'économie politique, la pensée bourgeoise posthégélienne se distingue immédiatement et brusquement (inconsciemment) de l'abstraction philosophisante par une sphère d'immédiateté sans concept, que ce soit l'existence ou la vie, qui fige de la sorte son irrationalité de façon apologétique. Mais même dans ce domaine de l'irrationalité immédiate d'une vie qui semble seulement être rationnelle mais ne l'est pas du tout, l'aspiration nostalgique [Sehnsucht] à la liberté et au bonheur se rabat sur une médiation entre la sensibilité et l'intelligibilité; exemplairement dans le médium de l'esthétique (cf. Adorno, Kierkegaard) (8).

La pensée de Nietzsche, sa devise de la vita periculosa est exemplaire : la décadence est la tentative de la société bourgeoise tardive, de traduire la tentative esthétique de la médiation historico-philosophique des mundus sensibilis et intelligibilis, en un médium qui n'est plus philosophique, à savoir celui de la vie vécue immédiatement. Le résultat de cette tentative de médiation immédiatement imposée, est la ruine existentielle du caractère intelligible, dont l'allégorie représente le caractère décadent (Baudelaire) (9). La vie en soi se transforme en une apparence [Schein] qui se sait apparence, et qui semble dissoudre toute réalité - une apparence qui n'est cependant pas celle qui est essentielle dans la raison réflexive hégélienne, mais une apparence qui se sait nonvérité effective - elle aussi ayant fait irruption immédiatement dans l'existence. La vie n'est qu'une apparence [Schein] parce que l'être [Sein] est devenu un non-vrai.

\section{De la décadence}

33 La décadence est le lieu empirique du caractère intelligible, aussi de sa souffrance, une pure allégorie (L'impérialisme et ses cris guerriers sont [illisible] de la décadence). Adorno : au sujet de la décadence et du progrès : «Le progrès signifie se libérer de l'emprise, aussi de l'emprise du progrès, qui est lui-même nature, lorsque l'humanité réintériorise sa propre naturalité et impose un arrêt à la domination qu'elle exerce sur la nature et par laquelle la domination de la nature se poursuit. À cet égard, on pourrait dire que le progrès a lieu là où il s'arrête. Cette image est cryptée dans un concept que tous les camps diffament aujourd'hui d'une seule voix, celui de la décadence » $(10)^{28}$.

34 La décadence a un sens relevant de la théorie de la connaissance dans une philosophie qui n'en est plus seulement une et qui aspire à se réaliser. Elle est la réflexion lourde de fatalité qui dévoile les médiations et des traînards incapables d'action, des Hamlets romantiques, celle qui produit ce degré de somnolence d'un peuple que Nietzsche condamnait et devant lequel il mettait en garde, qui est nuisible (le [illisible] de Hans Castorp, la musique [illisible]), retourné avec trop d'empressement dans la dénonciation existentielle du concept, l'apologie anti-théorique de la spontanéité de l'intuition non cognitive ou de la résolution existentielle.

35 La décadence est la réflexion qui est consciente de sa fragilité, pas seulement la réflexion de l'immédiateté aliénée de l'apparence [Schein] de son essence, mais celle de 
l'apparence qui se sait essence, l'apparence qui comprend et dévoile son essentialité et substantialité comme relevant de la société bourgeoisie tardive, comme une tromperie idéaliste. La fonction liée aux Lumières de la société bourgeoise tardive n'est pas sans impact positiviste: sa décadence tient d'abord (l'impact criminalistique) dans le fait que la société bourgeoise tardive dévoile et exprime ce que la bourgeoisie cachait jusqu'alors sous le couvert idéologique de la morale.

Le pathos des Lumières réside dans la soumission volontaire immoraliste à l'horreur (en ce sens, il dépasse le positivisme qui méconnait sa nature de soumission explicite au mythe), la souffrance, que le caractère décadent ne supporte cependant pas non plus. La décadence est la négation du progrès, qui postule le progrès dans sa négativité, la sensiblerie [Empfindlichkeit] exacerbée qui révèle l'horreur de la praxis quotidienne à laquelle les gens conformes [scil. aux rapports sociaux] ont été habitués. La décadence est l'extrême individuation. Le caractère allégorique de la décadence qui, comme Baudelaire, selon Benjamin, voit aussi le cadavre de l'intérieur (11).

\section{Décadence}

37 La décadence est l'extériorisation empirique du caractère intelligible, son cadavre social. Son expression esthétique est l'allégorie [toujours] enfermée sur elle-même qui est dirigée vers l'extérieur, la psychè du cadavre devenue physiquement immédiate [illisible] - c'est ce que Benjamin a trouvé et que Baudelaire a été le premier à articuler poétiquement.

\section{Notes et commentaires}

(1) Dans son essai sur Kafka, Benjamin associe les créatures difformes du monde réifié à l'oubli. L'emprise de la société redevenue seconde nature efface la mémoire de son avènement et produit les êtres maudits qui jaillissent des origines mythiques. Voir W. Benjamin, « Franz Kafka. Pour le dixième anniversaire de sa mort » (1934), in CEuvres II, traduit de l'allemand par Maurice de Gandillac, Pierre Rusch et Rainer Rochlitz, Paris, Gallimard, 2000, p. 439 : «L'époque où vit Kafka ne représente pour lui aucun progrès par rapport aux premiers commencements. Le monde où se déroulent ses romans est un marécage. La créature, chez lui, apparait à un stade que Bachofen désigne comme le stade hétaïrique. Que ce niveau d'existence soit oublié n'empêche pas qu'il se prolonge jusque dans le présent. Bien au contraire : c'est parce qu'il est oublié qu'il est présent »; et p. 441 : «Ce qui est oublié (...) n'est jamais d'ordre purement individuel. Tout ce que l'on oublie se mêle à la réalité oubliée du monde primitif, s'unit à elle par des liens innombrables, incertains, changeants, pour produire des fruits toujours nouveaux. L'oubli est, dans les récits de Kafka, le réservoir d'où surgit à la lumière l'inépuisable intermonde ». L'oubli frappe le monde livré aux puissances aveugles du capital et de la technique, qui renouent avec les puissances pré-mythiques de l'histoire des origines. Cette emprise ramène les êtres à des formes pré-humaines: "Comme le monde des faits importants, le monde des ancêtres s'étend pour Kafka à l'infini, et, comme les arbres totémiques chez les primitifs, plonge ses racines dans le règne animal. [Chez Kafka], les bêtes sont le réceptacle de ce qui est tombé dans l'oubli (...). Une chose est sûre : parmi toutes les créatures de Kafka, ce sont les bêtes qui réfléchissent le plus. La peur est dans leur pensée ce que la corruption est dans le droit. Elle gâche le déroulement de l'action, et elle est pourtant en celle-ci le seul élément qui recèle de 
l'espoir. Mais puisque nous habitons notre corps - notre propre corps - comme le pays étranger le plus oublié, on comprend que cette toux qui éclatait au fond de lui-même, Kafka l'ait appelée "la bête". Elle était le poste le plus avancé de la grande horde " (Ibid., p. 442-443). L'oubli et l'animalité sont deux manifestations de la rupture de la belle apparence dans laquelle le classicisme voit le triomphe de la liberté de l'humanité réalisée. Le monde de Kafka et le monde de l'allégorie représentent la non-liberté des êtres dans la non-réconciliation des scissions.

(2) «Das Leben lebt nicht» est l'énoncé programmatique des Minima Moralia de Theodor W. Adorno (traduction française de Jean-René Ladmiral et Eliane Kaufholz, Paris, Payot, 1980), repris à l'écrivain autrichien Ferdinand Kürnberger (1821-1879), qui avait dû s'exiler à Dresde suite à sa participation à la Révolution de 1848. La phrase est tirée du livre de 1855 Der Amerika-Müde, amerikanisches Kulturbild, que Max Weber mentionne dans ses études sur la recherche systématique du gain dans la culture américaine. Voir Max Weber, L'éthique protestante et l'esprit du capitalisme, édité, traduit et présenté par Jean-Pierre Grossein, Paris, Gallimard, 2003, p. 23-24 : «C'est Benjamin Franklin qui nous sermonne dans ces préceptes [portant sur la soumission de l'existence aux principes du gain et du calcul], ceux-là mêmes que Ferdinand Kürnberger donne pour la profession de foi du yankisme et qu'il raille dans son "tableau de la culture américaine", débordant d'esprit et de venin (...). Attardons-nous encore un peu sur ce passage, dont Le Fatigué de l'Amérique de Kürnberger résume ainsi la leçon de sagesse : "Avec les bovins on fait du suif, avec les humains, de l'argent" "; et p. 23, n. 26: «En tant qu'œuvre d'art, ce livre [Der Amerikamüde] serait aujourd'hui assez difficile à goûter, mais en tant que document sur les oppositions (aujourd'hui bien estompées) entre les sensibilités allemande et américaine (...) il n'a tout simplement pas été dépassé ». Le livre d'Adorno représente lui aussi une méditation sur la civilisation américaine et sur ses effets traumatiques sur la civilisation bourgeoise européenne : les Minima Moralia sont consacrés à la discipline philosophique traditionnelle qu'est la Doctrine de la vie bonne et à son impossibilité dans les conditions de la société capitaliste avancée incarnée par les États-Unis. La notion de "vie» est évoquée à plusieurs reprises dans l'ouvrage adornien: par son sous-titre Reflexionen aus dem beschädigten Leben ("Réflexions sur la vie mutilée ») et par un autre célèbre énoncé, «Es gibt kein richtiges Leben im falschen». Cette insistance sur la vie et sur son incomplétude, ses manques et ses mutilations recèle une double signification : Adorno veut dire que la « vie », la « vraie vie » qu'est la vie réconciliée, a été rendue impossible par le capitalisme ; mais il suggère aussi que toute tendance à ériger la Vie en positivité intacte et en réserve d'authenticité s'opposant aux conditions sociales aliénées est en fait une tendance régressive. Le fantasme de la plénitude vitale est tout aussi inacceptable que la capitulation face à la destruction de la subjectivité, dont la valeur ancienne consistait moins dans la communion avec une Vie mythifiée que dans la sobre dignité de l'existence bourgeoise classique. L'usage critique de l'esthétisme décadent, et par là des mutilations imposées à la vie, fait l'objet des notes de Krahl. La possibilité d'un tel usage suppose la rupture avec le vitalisme "positif » et l'assomption de la « vie» en tant que moment de la scission du sujet moderne. A ce sujet, voir aussi la critique par Benjamin du vitalisme métaphysique qui voudrait mettre la vie à la place du concept en tant que lieu et opérateur du dépassement des scissions, W. Benjamin, "Sur quelques thèmes baudelairiens ", in Cuvres III, traduit de l'allemand par Maurice de Gandillac, Pierre Rusch et Rainer Rochlitz, Paris, Gallimard, 2000, p. 331 : « Depuis la fin du siècle dernier, la philosophie a entrepris toute une série de tentatives pour 
ressaisir la "véritable" expérience, par opposition à celle qui se manifeste dans l'existence normalisée et dénaturée des masses soumises à la civilisation. On a coutume de classer ces essais sous la rubrique de la philosophie de la vie. On comprend qu'ils ne soient pas partis de l'existence sociale de l'homme. Ils ont invoqué la littérature, plus encore la nature et finalement la mythologie. Le livre de Dilthey, L'Expérience vécue et la littérature, est l'un des plus anciens dans une série qui conduit à Klages et à Jung, lequel a vendu l'âme au fascisme ».

(3) Krahl reprend ici les termes de Nietzsche : «La valeur de ce qu'il y a de plus bref, de plus périssable, le séduisant scintillement d'or sur le ventre du serpent vita" (Friedrich Nietzsche, Fragments posthumes, in Euvres philosophiques complètes, tome XIII, textes et variantes établis par Giorgio Colli et Mazzino Montinari, Paris, Gallimard, 1987, p. 26). Cette image représente la vie comme apparence et semblant - pur apparaître séparé du concept, de la valeur et de l'essence. La vie qui se sépare de la réalité substantielle et permanente en devenant un pur semblant correspond à la pensée qui devient une pure apparence esthétique en se séparant du concept, lequel vise la substance rationnelle de la réalité. Cette double séparation exprime une révolte contre la conciliation idéaliste entre pensée et réalité, entre être et paraître. Mais la séparation isolée débouche sur la construction d'une mythologie de l'expérience esthétique censée exprimer la pure et simple surface de l'existence, le pur et simple pouvoir créateur de la fiction en tant que manifestation vitale: par là, ce qui est manqué est précisément le rapport entre l'existence immédiate et la vérité que l'idéalisme avait cerné, bien que sous une forme insuffisante (cf. Babette Babich, « Nietzsche et Eros entre le gouffre de Charybde et l'écueil de Dieu : la valence érotique de l'art et l'artiste comme acteur-juif-femme", in Revue internationale de philosophie, $1 / 200, n^{\circ} 211$, p. 22 : «Pour comprendre le sens de ces valeurs [dont le serpent vita est métaphore], il est indispensable de souligner qu'elles sont valeurs de l'apparence, et qu'elles sont des valeurs sans (réelle) valeur : un reflet brillant doré, rien de plus qu'une lueur sur le ventre d'une métaphore. Seules la poésie ou la musique de l'invention artistique peuvent atteindre la suprême volonté de puissance »). Selon Krahl, c'est la praxis politique, et non la création esthétique, qui peut surmonter les apories de la conciliation idéaliste.

(4) La notion de Gattungswesen ou être générique désigne un concept fondamental dans la pensée de Ludwig Feuerbach et du jeune Marx (Karl Marx, Manuscrits économicophilosophiques de 1844, nouvelle traduction par Franck Fischbach avec présentation et notes, Paris, Vrin, 2007). Voir L. Feuerbach, Manifestes philosophiques, textes choisis et présentés par Louis Althusser, Paris, Puf, 1960, en particulier p. 6-7. À propos des deux termes qui composent ce concept - Gattung, le genre, et Wesen, l'essence - Althusser précise que : «Le terme de Wesen désigne tantôt l'être existant en chair et os, l'être réel, tantôt l'essence et la vérité intérieures de cet être (...). La Gattung n'est pas une simple catégorie biologique. Elle est une catégorie par essence théorique et pratique. Dans le cas privilégié de l'homme, elle apparaît à la fois comme le véritable horizon transcendantal qui permet la constitution de toute signification théorique, et comme l'Idée pratique qui donne son sens à l'histoire ". Le concept de Gattung est déjà présent dans les Lettres sur l'éducation esthétique de l'homme (1795) de Friedrich Schiller. Dans cet ouvrage qui constitue une transition décisive entre les Lumières allemandes et l'Idéalisme, Schiller qualifie le spectateur idéal de la Révolution française - le destinataire de cette interpellation par la Raison incarnée dans les événements politiques - d'« individu (...) capable de se placer au centre de l'univers et de se hausser 
au niveau de l'espèce humaine [wer nur immer fähig ist, sich in das Zentrum des Ganzen zu versetzen und sein Individuum zur Gattung zu steigern] ( Fr. Schiller, Lettres sur l'éducation esthétique de l'homme, traduites de l'allemand et préfacées par Robert Leroux, édition mise à jour par Michèle Halimi, Paris, Aubier, 1943, 1992, p. 88-89. Nous avons parfois modifié la traduction par souci de cohérence terminologique). Or dans la pensée de Schiller, l'art est précisément censé opérer cette unité entre l'individu et l'espèce qui devrait permettre de réaliser la Raison pure dans la situation post-révolutionnaire sans reproduire la Terreur. La triple articulation entre politique, esthétique et philosophie qu'abordent les notes de Krahl se confrontant avec Adorno, Benjamin et Lukács, caractérise toute la pensée classique allemande de Lessing à Marx.

(5) F. W. J. Schelling, Les Âges du monde, fragments dans les premières versions de 1811 et 1813 éditées par Manfred Schröter, traduit de l'Allemand par Pascal David, Paris, Puf, 1992. En dépit de l'allusion que Krahl fait aux Âges du monde, cet ouvrage inachevé est moins apte que d'autres textes de Schelling - tel le Système de l'idéalisme transcendantal à illustrer le souci schellingien de réconciliation entre le réel et l'idéal, le sensible et l'intelligible. Cependant, ce qui rend décisive la tentative des Âges $d u$ monde, est que Schelling essaie d'y articuler le logos et le réel prélogique au sein d'un processus historique de constitution de la conscience dont la scène originaire est l'irruption de la liberté dans l'opacité de la matière. Voir aussi le commentaire de Slavoj Žižek, Fragile absolu, traduit de l'anglais par François Théron, Paris, Flammarion, p. 105: «C'est précisément un récit mythopoïetique et fantasmatique qui rendrait compte de l'apparition du logos à partir du Réel protocosmique et prélogique que Schelling s'est efforcé d'accomplir dans les Weltalter. Mais à la fin de chacune des trois versions successives de cette œuvre - c'est-à-dire au point où aurait dû se déployer le passage du mythos au logos, du Réel au Symbolique -, Schelling a été obligé de postuler un acte bizarre d'Ent-Scheidung (une décision différenciative), un acte qui en un sens était plus originaire encore que le Réel du "passé éternel lui-même" ». Autrement dit, Schelling reconnaît la nature "impossible » de la médiation entre la pensée et l'être, entre la vie et le concept. L'échec de la construction systématique de cette médiation témoigne bien sûr de l'impuissance du concept à produire la réconciliation; mais il manifeste tout aussi bien l'insuffisance du recours au récit mythique, à l'intuition esthétique érigée en représentation mythologique. Ni le concept, ni la vie ne peuvent opérer la médiation effective car ils dépendent d'un médiateur impossible, abyssal, destiné à disparaître dans l'unité qu'il produit entre l'être et la pensée : cf. Schelling, op. cit., p. 213, cité par Žižek, op. cit., p. 106-107 : «De même, donc, que cet acte, une fois qu'il a eu lieu, sombre immédiatement dans une insondable profondeur, et adopte ce faisant la nature de ce qui est constant, il faut de même que cette volonté, une fois posée comme commencement et menée à l'extériorité, sombre immédiatement dans l'inconscience. C'est ainsi seulement qu'un commencement est possible, un commencement qui ne cesse plus d'être commencement véritablement éternel. Car ici aussi il convient de dire : le commencement n'a pas le droit de se connaître. Une fois accompli, cet acte est éternellement accompli. La décision qui, peu importe comment, doit constituer un commencement ne doit pas revenir à la conscience, elle ne doit pas être rappelée - ce qui revient presque à dire révoquée. Celui qui, en prenant une décision, se réserve le droit de la rappeler au jour, celui-là ne commence jamais ». Que la médiation relève d'un acte impossible, extérieur à la pensée et irréductible à toute plénitude ontologique de la Vie, permet donc de penser l'excès du réel sur le concept tout en déjouant la tentation de restaurer une mythologie de l'intuition. En ce sens, la position de Žižek 
semble pouvoir être rapprochée de celle de Krahl, qui assigne à l'action politique la tâche d'opérer la réconciliation que l'Idéalisme allemand a essayé de penser.

(6) Les Lettres de Schiller sont un texte décisif pour la problématique esthétique de la philosophie classique allemande et du marxisme occidental: l'activité esthétique y apparaît à la fois comme un opérateur politique majeur et comme un ersatz du politique. Le statut dialectique de l'esthétique à propos duquel Lukács et Adorno se confronteront, est déjà formulé par Schiller à partir du bilan de la Révolution française esquissé par les Lumières allemandes : «Le philosophe et l'homme du monde sont dans l'attente. Ils ont les yeux fixés sur le théâtre des événements politiques où le grandiose destin de l'humanité est, croit-on, en train d'être débattu. N'est-ce pas trahir une indifférence blâmable à l'égard du bien de la société que de ne pas participer à cette conversation [Gespräch] générale? Par son contenu et par ses conséquences cet important procès regarde quiconque revendique le nom d'homme ; et par la méthode, il doit intéresser quiconque pense par soi-même [jeden Selbstdenker]» (Fr. Schiller, Lettres sur l'éducation esthétique de l'homme, op. cit., p. 89). La " conversation » et le " penser par soi-même " sont deux concepts majeurs dans l'anthropologie politique des Lumières qu'incarne l'œuvre de Lessing. Le Gespräch est la réalisation concrète d'une sociabilité à la fois rationnelle et affective, et le Selbstdenken est l'opération d'une pensée autonome toujours déjà inscrite dans un espace de "conversation" (Voir à ce sujet Hannah Arendt, « De l'humanité dans des "sombres temps" » (1959), in Vies politiques, traduit de l'anglais et de l'allemand par E. Ada, J. Bontemps, B. Cassin, D. Don, A. Kohn, P. Lévy, A. Oppenheimer-Faure, Paris, Gallimard, 1974, p. 17-18: « [Lessing] n'a, comme il le savait lui-même, semé dans le monde "que des fermenta cognitionis". Ainsi, la célèbre Selbstdenken de Lessing -penser par soi-même - n'est en aucun cas l'activité d'un individu unitaire, clos, mûri, cultivé organiquement (...). Les fermenta cognitionis semés dans le monde par Lessing, n'étaient pas faits pour communiquer des connaissances, mais pour inciter les autres à penser par soi-même, et ce, sans autre dessein que de provoquer un dialogue entre penseurs. Le penser de Lessing n'est pas un parler avec soi-même mais l'anticipation d'un parler avec d'autres »). Ces deux concepts désignent l'ouverture et le développement d'un espace commun créé et reproduit par l'échange éclairé des idées et des affects. Les Lettres se présentent comme un paradigme de cet idéal du dialogue en tant que matrice de l'espace public proto-bourgeois : "Combien attrayant ne devrait-il pas être pour moi d'aborder l'étude d'un pareil sujet et de le débattre avec un homme qui est un penseur plein d'esprit autant que caractère libéral et citoyen du monde, et d'en confier la solution à un cœur qui se consacre avec un bel enthousiasme au bien de l'humanité! " (Fr. Schiller, Lettres sur l'éducation esthétique de l'homme, op.cit., p. 91). Pourtant, Schiller affirme d'emblée l'impossibilité de traiter directement de ce «sujet " capital - l'irruption de la raison dans la vie historicopolitique. La conversation devra faire un détour avant de pouvoir aborder les questions politiques, et portera d'abord sur des questions esthétiques: "Si je résiste à cette séduisante tentation et si je fais passer la Beauté avant la liberté, je crois non seulement excuser cette méthode par une inclination personnelle, mais encore la justifier par des principes (...). Pour résoudre dans l'expérience le problème politique dont j'ai parlé, la voie à suivre est de considérer d'abord le problème esthétique ; car c'est par la beauté que l'on s'achemine à la liberté » (Ibid.). La médiation esthétique est nécessaire si l'État authentiquement rationnel doit pouvoir réconcilier les antinomies du sensible et de l'intelligible, du multiple et de l'unité : «La Raison certes réclame de l'unité, mais la nature demande de la multiplicité, et l'homme est sollicité par ces deux législations (...). 
Une Constitution sera très incomplète si elle n'est capable de produire l'unité qu'en supprimant la multiplicité. L'État ne doit pas honorer dans les individus seulement leur caractère objectif et générique, mais encore leur caractère subjectif et spécifique " (Ibid., p. 105); "Lorsque donc la raison introduit son unité morale dans la société physique, elle n'a pas le droit de porter atteinte à la multiplicité de la nature. Lorsque la nature aspire à affirmer sa multiplicité dans l'édifice moral de la société, il ne faut pas que l'unité morale en éprouve un dommage quelconque; la Forme victorieuse est à égale distance de l'uniformité et du désordre. Il faut donc qu'un peuple possède un caractère total [Totalität des Charakters] pour qu'il soit capable et digne d'échanger l'État de la nécessité contre l'État de la liberté » (Ibid., p. 109). La beauté est l'opérateur de cette unité organique des facultés humaines. La Sixième lettre expose les raisons de l'impossibilité pour l'homme contemporain de réaliser cette réconciliation. La scission dont la subjectivité moderne est porteuse rend vain tout espoir de donner une forme concrète à l'idéal de l'État rationnel : «Si nous considérons avec quelque attention le caractère de notre temps, nous ne pouvons qu'être étonnés par le contraste que nous observons entre la forme actuelle de l'humanité et celle des hommes de l'antiquité, notamment des Grecs (...). Au temps où se produisit ce magnifique éveil des facultés de l'âme, les sens et l'esprit n'avaient pas encore des territoires strictement séparés ; aucune scission [Zwiespalt] ne les avait encore excités à délimiter, dans un esprit d'hostilité, leurs domaines réciproques et à établir entre eux des frontières (...). Comme il en va tout autrement chez nous autres modernes! Chez nous aussi l'image de l'espèce se réfracte, agrandie, entre les individus; mais elle est fragmentée, non insérée de multiples façons en eux; en sorte que pour recomposer la totalité de l'espèce, il faut aller d'un individu à un autre et s'enquérir à la ronde (...); et nous voyons non seulement des individus mais encore des classes entières d'hommes ne déployer qu'une partie de leurs dispositions, tandis que leurs autres virtualités sont, comme dans des plantes rabougries, à peine indiquées par de faibles traces " (Ibid., p. 119-121). Cette scission oppose l'individu à l'espèce: alors même que les puissances collectives de l'humanité se développent comme une sphère impersonnelle, les capacités des individus et leur liberté se rétrécissent. Schiller indique les causes de cette condition déchirée: "D'où vient que les individus se trouvent ainsi désavantagés alors que l'espèce a l'avantage ? (...) Ce fut la civilisation elle-même qui infligea cette blessure à l'humanité moderne. Dès que d'un côté une séparation plus stricte des sciences, et de l'autre une division plus rigoureuse des classes sociales et des tâches furent rendues nécessaires (...) le faisceau intérieur de la nature humaine se dissocia lui aussi et une lutte funeste divisa l'harmonie de ses forces » (Ibid., p. 121-123). L'Aufklärer Schiller voit très clairement que l'avènement des conditions de vie modernes que préconise le programme des Lumières implique une mutilation de l'homme qui rend impossible l'accomplissement de ce programme: «Ce bouleversement que l'artifice de la civilisation et la science commencèrent à produire dans l'homme intérieur, le nouvel esprit des gouvernements le rendit complet et universel (...). Les États grecs, où, comme dans un organisme de l'espèce des polypes, chaque individu jouissait d'une vie indépendante, mais était cependant capable, en cas de nécessité, de s'élever à l'Idée de la collectivité, firent place à un ingénieux agencement d'horloge dans lequel une vie mécanique est créée par un assemblage de pièces innombrables mais inertes (...). L'homme qui n'est plus lié par son activité professionnelle qu'à un petit fragment isolé $\mathrm{du}$ Tout ne se donne qu'une formation fragmentaire; n'ayant éternellement dans l'oreille que le bruit monotone de la roue qu'il fait tourner, il ne développe jamais 
l'harmonie de son être, et au lieu d'imprimer à sa nature la marque de l'humanité, il n'est plus qu'un reflet de sa profession, de sa science (...). La communauté sociale fait de la fonction le critère de l'homme; elle n'honore chez tel de ses citoyens que la mémoire, chez tel autre que l'intelligence de tabellion, chez un troisième que l'aptitude mécanique [...]; ces capacités isolées, elle désire qu'en même temps l'individu les développe en gagnant en intensité ce qu'elle lui permet de perdre en étendue. Comment s'étonner alors que l'on néglige les autres dispositions de l'âme pour consacrer tous ses soins à celle qui seule procure honneur et profit ? (...) Ainsi peu à peu la vie concrète des individus est-elle abolie afin de permettre à la totalité abstraite de persévérer dans son indigente existence, et l'État, la société positive se dissout (c'est depuis longtemps déjà le destin de la plupart des nations européennes) et tombe du point de vue de mœurs dans l'état de nature [Naturstand]» (Ibid., p. 123-127). Le projet schillerien consistera donc à reconstituer la totalité perdue : «Il doit être en notre pouvoir de rétablir dans notre nature la totalité que l'artifice de la civilisation a détruite, de la restaurer par un art supérieur » (Ibid., p. 135). Mais cet " art supérieur » exclut d'emblée la politique proprement dite: «Si l'expérience confirme mon tableau du temps présent, on doit proclamer inopportun tout essai d'opérer une pareille transformation de l'État, et chimérique toute espérance fondée sur une pareille tentative; il en sera ainsi aussi longtemps que le déchirement de l'homme intérieur n'aura pas été supprimé et que sa nature n'aura pas été si complètement développée qu'elle puisse devenir l'ouvrière de cette œuvre et garantir la réalité de l'édifice politique créé par la Raison» (Ibid., p.137-139). L'art supérieur qui pourra guérir l'humanité blessée est la pratique esthétique: "Toute amélioration dans l'ordre politique doit partir de l'ennoblissement du caractère (...). L'instrument recherché est le bel art; ses modèles immortels sont les sources qui s'ouvrent à nous » (Ibid., p. 149). Pour Schiller, les paradigmes de l'harmonie et de la liberté qu'incarnent les formes artistiques réalisent dans la «belle apparence » ce qui ne saurait être effectué dans la réalité historique : «L'humanité a perdu sa dignité, mais l'art l'a sauvée et conservée dans des pierres pleines de sens; la vérité continue à vivre dans l'illusion, et l'original sera reconstruit à partir de la copie [aus dem Nachbilde wird das Urbild wieder hergestellt werden]» (Ibid., p. 153).

Le programme formulé par Schiller aura des conséquences incalculables. Sa critique de la société moderne retentira jusque dans la Philosophie du droit de Hegel, la critique marxienne de l'économie politique et le diagnostic à propos de la rationalisation moderne en tant que «cage d'acier» chez Max Weber. Ces trois sources alimenteront ensuite la problématique du « marxisme occidental » développée par Lukács, Benjamin, Adorno, Marcuse... Chez ces auteurs, on retrouve la figure conceptuelle capitale de l'art, considéré à la fois comme sphère décisive du point de vue politique et comme ersatz d'une politique pour laquelle les conditions de réalisation ne sont pas données. C'est à partir de cette tension qu'on peut situer les apories du marxisme occidental et les tentatives de les dépasser en direction d'une politisation plus directe de ses problématiques.

(7) Allusion au texte de G. E. Lessing, Die Erziehung des Menschengeschlechts (1780). Cet ouvrage d'un des plus importants auteurs des Lumières allemandes anticipe sur les philosophies dialectiques de l'histoire de l'Idéalisme en prenant pour objet le processus de la prise de conscience dans l'histoire en tant que médiation entre l'individu et le genre humain: « $\$ 1$. La révélation est au genre humain ce que l'éducation est à l'individu. § 2. L'éducation est la forme de révélation qui est donnée à l'individu; la 
révélation est la forme d'éducation qui a été donnée au genre humain et qui continue à l'être.» (G. E. Lessing, L'éducation du genre humain, précédé de Dialogues maçonniques, introduction, traduction et notes par Pierre Grappin, Paris, Aubier-Montaigne, 1968, p. 91). Comme on l'a rappelé plus haut, la pensée de Lessing a exercé une très grande influence sur les Lettres de Schiller.

(8) Theodor Wiesengrund Adorno, Kierkegaard. Konstruktion des Ästhetischen (1933), traduit de l'allemand par Éliane Escoubas, Paris, Payot, 1995. L'étude d'Adorno propose une critique du mythe bourgeois-tardif de l'intériorité, dont la bourgeoisie du XXe siècle voudrait faire un espace d'authenticité, et qui constitue pour l'auteur le lieu du surgissement des puissances mythiques auxquelles est livré l'individu isolé dans un monde réifié. Dans un compte-rendu de 1933, Benjamin écrit : «L'idéalisme esthétique propre au romantisme fait apparaitre au grand jour les aspects mythiques de l'idéalisme absolu (...). Kierkegaard révèle "dans le déclin historique de l'idéalisme", comme le sens originel de cet idéalisme même, "un contenu mythique qui est en même temps historique" (...). Ainsi, l'intériorité kierkegaardienne se voit assigner un lieu déterminé dans l'histoire et dans la société. Son modèle est l'intérieur bourgeois dans lequel se confondent des traits historiques et mythiques » (W. Benjamin, « Kierkegaard. La fin de l'idéalisme philosophique ", in Eeuvres II, traduit de l'allemand par Maurice de Gandillac, Pierre Rusch et Rainer Rochlitz, Paris, Gallimard, 2000, p. 356-357). Dans la même recension, une remarque de Benjamin sur la méthode d'Adorno reformule encore le problème de (l'insuffisance de) la médiation esthétique comme opérateur de la réconciliation dialectique: "Wiesengrund a emprunté à l'œuvre de Kierkegaard un certain nombre de descriptions fascinantes d'espaces intérieurs de ce type. Dans ces passages, l'intériorité se révèle "la prison historique de l'humanité primitive" (...). Jamais Wiesengrund n'est plus profond que là où, négligeant les schémas de la philosophie kierkegaardienne, il en cherche la clé dans ses résidus les moins voyants : ses images, ses paraboles, ses allégories. Le mouvement, dont parlent les contes chinois, de la disparition (du peintre) dans le tableau (qu'il a lui-même peint) nous apparaît comme le dernier mot de cette philosophie. "Disparaissant, [le soi] est sauvé par son rétrécissement". Une telle absorption par le tableau n'est pas rédemption mais consolation. Consolation dont la source est l'imagination "en tant qu'organon d'une transition continue qui conduit du mythico-historique à la réconciliation" " (Ibid., $p$. 357-358). Par ce travail sur les aspects marginaux et mineurs de l'écriture kierkegaardienne, Adorno vise à produire un mouvement esthético-dialectique par lequel l'emprise des puissances mythiques est dissoute dans le processus immanent et critique de la pensée. L'imagination qui s'exerce sur des images historico-conceptuelles est l'opérateur de cette pensée et la condition du dépassement immanent de l'idéalisme esthétique: par là, la stratégie d'Adorno reste interne à l'idée d'une conciliation esthético-spéculative de la vie mutilée, qui relève entièrement de l'horizon romantique et idéaliste, et à propos de laquelle Benjamin était peut-être plus sceptique que son disciple. C'est pourquoi, il insiste sur la différence entre rédemption et consolation : la critique immanente de l'apparence ne représente qu'une solution spéculative, une apparence de réconciliation - tandis que la véritable rédemption aurait fini par se rapprocher, chez Benjamin, de la sphère politique. Dans les fragments de Krahl aussi, c'est la pratique politique, et non la théorie esthétique critico-dialectique, qui peut surmonter la crise de l'idéalisme.

(9) Krahl avance donc que, lorsque la vie immédiate est chargée de réaliser la médiation entre sensible et intelligible, réel et idéal, la vie elle-même finit par se déréaliser, et le 
monde se présente sous la forme de l'allégorie. L'abstraction mortifère de l'existence capitaliste est la base des analyses que Benjamin consacre à l'allégorie dans «Paris, capitale du XIXe siècle », in Euvres III, traduit de l'allemand par Maurice de Gandillac, Pierre Rusch et Rainer Rochlitz, Paris, Gallimard, 2000, p. 58-59: «Le génie de Baudelaire, nourri de mélancolie, est un génie allégorique. Avec Baudelaire, Paris devient pour la première fois un objet de la poésie lyrique. Cette poésie n'est pas un art local, le regard que l'allégoriste pose sur la ville est au contraire le regard du dépaysé. C'est le regard du flâneur, dont le mode de vie couvre encore d'un éclat apaisant la désolation à laquelle sera bientôt voué l'habitant des grandes villes. Le flâneur se tient encore sur le seuil, celui de la grande ville comme celui de la classe bourgeoise. Aucune des deux ne l'a encore subjugué. Il n'est chez lui ni dans l'une ni dans l'autre. Il se cherche un asile dans la foule. On trouve chez Engels et chez Poe les premières contributions à une physiognomonie de la foule. Celle-ci est le voile à travers lequel la ville familière apparaît comme fantasmagorie et fait signe au flâneur ». Benjamin insiste encore sur l'écart, et l'opposition, entre la position de la vie excentrique du décadent vis-à-vis de la société capitaliste et celle de la politique révolutionnaire: «Dans la personne du flâneur, l'intelligence va au marché. Pour en contempler le spectacle, croit-elle, mais, en vérité - pour y trouver un acheteur. A ce stade intermédiaire où elle a encore des mécènes, mais déjà commence à se familiariser avec le marché, elle se présente comme bohème. Au flou de sa situation économique correspond le flou de sa fonction politique. Laquelle apparait de la façon la plus visible chez les conspirateurs professionnels, qui tous viennent de la bohème. Leur premier champ d'action est l'armée, puis la petite bourgeoisie, occasionnellement le prolétariat. C'est pourtant parmi les véritables chefs du prolétariat que cette couche sociale trouve ses adversaires. Le Manifeste communiste met fin à son existence politique. La poésie de Baudelaire tire sa force du pathos de la rébellion que cultivent ces groupes » (Ibid., $p$. 59). Dans ses réflexions politico-philosophiques, Krahl s'interrogera sans cesse sur les conditions d'une révolte subjective qui ne reproduirait pas au sein du mouvement antiautoritaire la position de la subjectivité décadente.

(10) Adorno fait sans doute allusion ici aux positions esthétiques de Lukács et à sa polémique contre la décadence idéologique et artistique de la culture bourgeoise. Voir à ce propos Th. W. Adorno, "Une réconciliation extorquée ", in Notes sur la littérature, traduit de l'allemand par Sybille Muller, Paris, Flammarion, 1984, p. 175 : «On rejette l'ensemble de la littérature moderne tant qu'on ne peut pas lui appliquer la formule d'un réalisme critique ou socialiste, peu importe, et on lui attribue sans hésitation la marque infamante de la "décadence", une insulte qui sert à couvrir toutes les infamies de la persécution et de l'extermination, et pas seulement en Russie (...). Le discours sur la décadence est à peu près inséparable de l'image positive qui lui est opposée, celle de la nature débordante de vigueur ; c'est la projection de catégories naturelles sur ce qui est socialement médiatisé ». De cette polémique finalement assez malhonnête, on retiendra le refus opposé une fois de plus à toute valorisation de la positivité et de la plénitude vitales: pour Adorno, les formes esthétiques que Lukács assigne à la décadence manifestent la libération, et peut-être encore plus la protestation, de la particularité non-réconciliée, contre laquelle Lukács invoque le primat d'une totalité du sens et de la valeur. Selon Adorno, les tentatives de présenter une totalité réalisée ne sauraient être que des mystifications, tant à l'Est qu'à l'Ouest. La reformulation par Krahl de ce différend introduit un déplacement de problématique par rapport aux deux maitres de l'esthétique dialectique, dans la mesure où la fonction critique de 
l'esthétique "décadente » est à la fois affirmée et opposée à la relève politique des apories des médiations bourgeoises : c'est pourquoi Krahl peut à la fois accepter, contre Lukács, que l'autonomisation du particulier puisse revêtir une fonction critique vis-àvis des conciliations mystifiées, et utiliser, contre Adorno, le mot « décadence » pour indiquer les limites d'une position critique qui reste dans le champ impolitique de l'esthétique, voire de l'esthétisme.

(11) L'allégorie est le principe de la figuration esthétique du monde de la décadence. La position décadente vis-à-vis du monde réifié est manifestée par l'allégorie, dont le principe formel exprime les scissions de la réalité non-réconciliée. Dans les théories esthétiques de la pensée classique allemande, l'allégorie s'oppose au symbole dans la mesure où celui-ci vise la réalisation esthétique d'une totalité organique et harmonieuse. Voir Goethe, Maximes et réflexions, traduit de l'allemand et présenté par Pierre Deshusses, Paris, Rivages, 2001, p. 85-86: «Le symbole transforme le phénomène en idée, l'idée en image, de sorte que dans l'image l'idée reste infiniment agissante et inaccessible (...). L'allégorie transforme le phénomène en concept, le concept en image, mais de façon telle que dans l'image le concept reste toujours limité et reconnaissable par elle (...). Il y a une grande différence entre un poète qui cherche le particulier pour aller vers le général et celui qui regarde ce qu'il y a de général dans le particulier. La première démarche produit l'allégorie où le particulier sert seulement d'exemple, d'illustration du général ; par contre la seconde est en fait la nature même de la poésie, elle énonce un particulier sans penser au général ou même y renvoyer. Celui qui saisit ce particulier dans ce qu'il a de vivant recueille en même temps le général sans s'en rendre compte ou simplement après coup». Goethe reproche corollairement à l'allégorie de briser l'unité vivante du particulier et du général, du réel et de l'idéal, du sensible et de l'intelligible, que le symbole présente comme réconciliés dans l'immédiateté de la belle forme: "L'art est un médiateur de l'indicible; c'est donc apparemment une folie que de vouloir s'en faire à son tour le médiateur par des mots (...). En esthétique, il n'est pas bienvenu de parler de l'idée du Beau, alors qu'il ne peut être pensé de façon isolée. On peut avoir une notion du Beau, et cette dernière peut être transmise (...). Le Beau nécessite une loi qui se manifeste dans l'apparence (...). Lorsque la loi se manifeste dans sa plus grande liberté et selon ses conditions propres, elle produit le Beau objectif qui nécessite toutefois des sujets dignes de l'appréhender " (Ibid., p. 82-84). Les positions esthétiques de Goethe se rapprochent de celles de Schiller, qui assignait à la médiation entre réel et idéal la tâche politique de la création de l'homme harmonieux. Le symbole classiciste est l'opérateur d'une réconciliation des scissions dans une totalité organique dont le statut est à la fois spéculatif, esthétique et politique. Ces passages de Goethe seront repris tant par Lukács que par Benjamin, dans leurs positionnements respectifs vis-à-vis de l'art d'avant-garde. Tandis que Lukács épouse les positions esthétiques de Goethe, Benjamin a entrepris une réévaluation de l'allégorie en insistant sur sa critique implicite de la réconciliation classiciste: «Le classicisme tend à l'apothéose de l'existence dans l'individu dont la perfection n'est pas seulement morale (...). Le rayon d'action de cet individu beau, achevé (...) décrit le cercle du "symbolique". À l'opposé, l'apothéose baroque est dialectique. Elle s'accomplit dans la transformation des extrêmes. L'intériorité non contradictoire du classicisme ne joue aucun rôle dans ce mouvement dialectique excentrique (...). En même temps que le concept profane de symbole du classicisme se développe sa réplique spéculative, l'allégorie ». (W. Benjamin, Origine du drame baroque allemand (1925), traduit de l'allemand par Sybille Muller et André Hirt, Paris, Flammarion, 1985, p. 218-219). 
Dans l'Origine du drame baroque allemand, Benjamin utilise les spéculations sur le symbolisme de l'érudit romantique Friedrich Creuzer pour fonder un mode d'expression où l'unité immédiate entre le particulier et l'universel, entre l'idée et le phénomène, est systématiquement brisée : "Le symbole "exige (...) la clarté (...) la brièveté (...) l'aimable et le beau"; la première et les deux dernières de ces exigences expriment distinctement une conception que Creuzer partage avec les théoriciens du symbole de la période classique (...). Il ne fait aucun doute que sur ce point l'admiration que Winckelmann vouait à la sculpture grecque, dont les statues de dieux lui servent d'exemples, fut déterminante pour Creuzer. Le symbole artistique est plastique. C'est l'esprit de Winckelmann qui s'exprime chez Creuzer dans l'antithèse du symbole mystique. "C'est ici l'empire de l'ineffable qui, cherchant à s'exprimer, brisera finalement la forme terrestre comme un vase trop fragile, par la puissance infinie de son être. Mais par là, la clarté de la vision est aussitôt anéantie, et il ne reste plus qu'une stupeur muette". Dans le symbole plastique, "l'être ne tend pas vers l'excès, mais, obéissant à la nature, il se plie à sa forme, la pénètre et l'anime. La contradiction entre le fini et l'infini est donc résolue, du fait que, s'imposant à lui-même ses limites, il se fait humain. Cette purification de la forme plastique d'une part, ce renoncement volontaire à l'incommensurable d'autre part, produisent le plus beau fruit de tout l'ordre symbolique. C'est le symbole des dieux, qui unit merveilleusement la beauté de la forme à la plénitude suprême de l'être, et parce qu'il connaît sa plus grande perfection dans la sculpture grecque, on peut lui donner le nom de symbole plastique". Le classicisme recherchait "l'humain" comme "plénitude de l'être", et (...) devait obligatoirement mépriser l'allégorie " (Ibid., p. 224-225. Benjamin tire les citations de Creuzer de l'ouvrage célèbre Symbolik und Mythologie der alten Völker, besonders der Griechen. 1. Theil, 2. völlig umgerab. Ausg., Leipzig, Darmstadt, 1819). Les travaux de Creuzer permettent de reconnaître la consistance et l'autonomie de formes d'expression qui rompent avec la plénitude du symbole classique, et qui peuvent par conséquent figurer les scissions d'un monde déchiré. L'opposition entre symbole et allégorie correspond à l'opposition entre deux formes de l'expérience du monde, deux voies qui mènent à la réconciliation : "Le rapport entre le symbole et l'allégorie peut être défini et formulé avec précision sous la catégorie décisive du temps, que la grande intuition romantique (...) a fait entrer dans le domaine de la sémiotique. Alors que dans le symbole, par la sublimation de la chute, le visage transfiguré de la nature se révèle fugitivement dans la lumière du salut, en revanche, dans l'allégorie, c'est la facies hippocratica de l'histoire qui s'offre au regard du spectateur comme un paysage primitif pétrifié. L'histoire, dans ce qu'elle a toujours eu d'intempestif, de douloureux, d'imparfait, s'inscrit dans un visage - non : dans une tête de mort. Et aussi vrai qu'il n'y a en celle-ci nulle liberté "symbolique" de l'expression, nulle harmonie classique de la forme, nulle humanité, l'énigme qui s'exprime dans cette figure, la plus soumise à l'empire de la nature, ce n'est pas simplement la nature de l'existence humaine, mais l'historicité de la biographie individuelle. C'est là le noyau de la vision allégorique, de l'exposition baroque de l'histoire comme histoire des souffrances du monde; elle n'a de signification que dans les stations de sa décadence. Autant de sens, autant d'emprise de la mort, parce que la mort enfouit au plus profond la ligne de démarcation brisée qui sépare la physis et la signification. Mais si la nature a de tout temps été gouvernée par la mort, elle a toujours été allégorique. La signification et la mort sont autant les produits du développement de l'histoire que des germes, imbriqués l'un dans l'autre, dans l'état de péché de la créature exclue de la grâce » (Ibid., p. 227). La réconciliation 
que promet l'allégorie implique donc l'exploration radicale du monde non réconcilié, tandis que le symbole vise à montrer la réconciliation comme toujours-déjà présente dans la totalité d'une apothéose humaniste. Adorno a élevé l'allégorie au rang de principe général de la fonction dialectique des œuvres esthétiques. Pour lui, la nonréconciliation entre l'Idée et l'apparence dans les poèmes de Hölderlin incarne une opposition au classicisme de Goethe et exprime l'indocilité du particulier face à la fausse conciliation: "La perte est entrée dans le concept et l'arrache au fade idéal de l'humain universel (...). L'histoire tranche le lien qui selon l'esthétique classique devait unir l'idée et la vision intuitive (...). [Goethe et Schiller ont subodoré] chez Hölderlin une antipathie contre l'harmonie esthétique du fini et de l'infini (...). En faisant voler en éclats l'unité symbolique de l'œuvre d'art, [Hölderlin] rappelle ce qu'il y a de mensonger dans la réconciliation de l'universel et du particulier au sein de l'irréconcilié ». (Theodor W. Adorno, "Parataxe », in Notes sur la littérature, op. cit., $p$. 325-327)

\section{NOTES}

1. Voir Séminaire du GRM du 13 novembre 2010, http://f.hypotheses.org/wp-content/blogs.dir/ 1106/files/2013/01/GRM4.LENINE.Krahl_.Cavazzini.pdf.

2. Le texte de Hans-Jürgen Krahl a été traduit et annoté par Andrea Cavazzini et Jérémy Hamers. Cette traduction a été réalisée à partir de la retranscription des notes originales de Hans-Jürgen Krahl conservées aujourd'hui au Verlag Neue Kritik. Cette traduction est dès lors tributaire des choix opérés par les auteurs de la retranscription et des mentions d'illisibilité signalées dans la retranscription disponible en ligne. La traduction française proposée ici résulte à son tour d'une série de choix terminologiques dont certains sont apparus nécessaires mais discutables aux traducteurs. Dans ces cas, le terme original allemand est mentionné dans le corps du texte. [Source : http://www.krahl-briefe.de/]

3. Le 9 octobre 2013, ce texte a fait l'objet d'un exposé intitulé « Esthétique et dialectique I. Les débats dans le marxisme occidental (1930-1960)» dans le Séminaire "Esthétique et Théorie critique » organisé par Maud Hagelstein à l'Université de Liège.

4. Sur le marxisme occidental et la centralité de l'esthétique dans sa problématique, voir Perry Anderson, Les origines de la postmodernité (1998), Paris, Les prairies ordinaires, 2010, p. 97-98 et A. Cavazzini, Le bon usage des ruines. Franco Fortini et la question des intellectuels dans la Séquence rouge italienne, in Cahiers du GRM II, La Séquence rouge italienne, EurophilosophieEditions, http:// grm.revues.org/230.

5. Au début des années 1960, Hans-Magnus Enzensberger adopte des positions analogues vis-à-vis de la critique pratique de l'industrie culturelle. On trouvera d'autres paradigmes de cette orientation dans la Nuova Sinistra italienne (cf. Andrea Cavazzini, « Notes sur la Théorie critique et l'industrie culturelle ", in Cahiers du GRM IV. Des luttes étudiantes en Europe occidentale 2, http:// grm.revues.org/310).

6. G. Lukács, « Marx et le problème de la décadence idéologique » (1938), in Problèmes du réalisme, traduit de l'allemand par Cl. Prévost et J. Guégan, Paris, L'Arche, 1975, p. 176.

7. G. Lukács, «L'idéal de l'homme harmonieux dans l'esthétique bourgeoise » (1938), in Problèmes du réalisme, op. cit., p. 230. 
8. Ibid., p. 231-232.

9. Ibid., p. 233-235.

10. Ibid., p. 236.

11. Ibid., p. 238.

12. Ibid.

13. Ibid.

14. Ibid., p. 241.

15. Franco Fortini faisait remarquer à juste titre que «Lukács ne renonça jamais à une position

(...) germanocentrique. La classe ouvrière est l'héritière des Universités; et le fonctionnaire soviétique du premier Plan Quinquennal remplace pour lui la classe ouvrière soviétique (entretemps disparue) : c'est pour ce fonctionnaire qu'il faut rouvrir la voie qui mène de Lénine à Marx et de Marx à Hegel » (F. Fortini, «Mandato degli scrittori e fine dell'antifascismo ", in Verifica dei poteri, Turin, Einaudi, 1965, 1989, p. 110).

16. G. Lukács, "Marx et le problème de la décadence idéologique » (1938), in Problèmes du réalisme, op. cit., p. 187-188.

17. Ibid., p. 196.

18. Ibid., p. 192.

19. Ibid., p. 211.

20. Th. W. Adorno-M. Horkheimer, Dialectique de la raison, traduction française par E. Kaufholz de Dialektik de l'Aufklärung, Paris, Gallimard, 1974, p. 139-140.

21. F. Fortini, «Mandato degli scrittori e fine dell'antifascismo», op. cit., p. 111. L'assassinat du dirigeant bolchevik Sergueï Kirov - très populaire dans le Parti et la classe ouvrière - fut le prétexte qui permit à Staline de déclencher les Grandes Purges en URSS.

22. Dans « Sur le concept d'histoire » (1940), Walter Benjamin avait bien cerné cet enracinement des objectivations culturelles dans la longue durée de "vertus » dont la Révolution elle-même aurait le plus grand besoin : les « choses raffinées et spirituelles » qui n'ont de cesse de témoigner de la barbarie des sociétés divisées en classes « interviennent dans la lutte de classe autrement que comme l'idée d'un butin qu'emportera le vainqueur. Comme confiance, courage, humour, ruse, fermeté inébranlable, elles prennent une part vivante à la lutte et agissent rétrospectivement dans les profondeurs du temps. Elles remettront toujours en question chaque nouvelle victoire des maîtres " (in CEuvres III, traduit de l'allemand par M. de Gandillac, Pierre Rusch et Rainer Rochlitz, Paris, Gallimard, 2000, p. 430). Dans ce passage, Benjamin reformule des positions qu'il avait déjà exposées dans son commentaire du poème de Brecht «Légende sur l'origine du livre Tao Te-king » : elles impliquent de fonder l'agir dans l'ici-et-maintenant sur des strates temporelles lentes et profondes. Mais ces strates sont celles que la poésie interroge depuis sa naissance. En 1921, Ossip Mandelstam avait écrit : «La poésie est la charrue qui affouille le temps afin d'en faire émerger les couches profondes (...). Or à certaines époques l'humanité, insatisfaite du quotidien, vient à se languir des couches profondes du temps et aspire, comme le laboureur, aux terres vierges du temps » (O. Mandelstam, "Verbe et culture », in De la poésie, traduit du russe, présenté et annoté par Mayelasveta, Paris, Gallimard, 1990, p. 46). Dans ce texte, Mandelstam appelait de ses vœux une séparation entre l'État et la culture, chacun obéissant à des ordres temporels différents. La disjonction entre la politique et la culture est un problème majeur $\mathrm{du}$ XXe siècle, qui refait surface dans les conjonctures les plus complexes et tragiques, telles celles dans lesquelles écrivaient Lukács, Brecht, Benjamin et Mandelstam.

23. H.-M. Enzensberger, «La littérature comme institution ou l'effet Alka-Seltzer », in Médiocrité et folie, Paris, Gallimard, 1991, p. 62.

24. Ibid., p. 61.

25. L'auteur utilise le terme « Bewandtnis ». On doit sans doute lire «Verwandtnis » qui pourrait être un néologisme déduit de "verwandt», c'est-à-dire "apparenté. » Le qualificatif "bewandert », soit « compétent ", « bien informé dans un domaine », pourrait également être à 
l'origine de ce néologisme. Mais il nous semble que cette seconde interprétation est inadéquate ici.

26. «Histoire de l'art » ne renvoie pas ici à la discipline. Krahl écrit « Geschichtsphilosophie der Kunst » et non «Philosophie der Kunstgeschichte ».

27. Le terme «mémoires » traduit ici «Erinnerungen ». Dans la phrase précédente tout comme dans la parenthèse qui suit, le terme «Erinnerung" (au singulier) est traduit par « remémoration » pour en rendre le sens plus actif («l'acte de se remémorer quelque chose »).

28. Theodor W. Adorno, "Fortschritt", in Gesammelte Schriften, Band 10.2, Frankfurt a. M., Suhrkamp, 1997, p.625. Par souci de cohérence avec le texte de Hans-Jürgen Krahl, nous proposons ici notre propre traduction de la citation d'Adorno. Pour une traduction complète du texte cité, voir : Th. W. Adorno, « Le progrès ", in Modèles critiques, traduit de l'allemand par Marc Jimenez et Éliane Kaufholz, Payot, Paris, 1984, 2003, p. 185-186.

\section{RÉSUMÉS}

L'article étudie - à partir des fragments de H.-J. Krahl - les débats sur l'esthétique et ses rapports avec la politique et la philosophie de l'histoire depuis la philosophie classique allemande jusqu'aux analyses post-adorniennes de l'industrie culturelle.

\section{INDEX}

Thèmes : esthétique, théorie critique, marxisme, philosophie allemande Index géographique : Allemagne

Mots-clés : dialectique, esthétique, politique, décadence, Krahl Hans-Jürgen Index chronologique : XVIII-XIX-XXe siècle

\section{AUTEURS}

\section{ANDREA CAVAZZINI}

Andrea Cavazzini fait activité de recherche à l'Université de Liège, il est membre du GRM et de l'Association « Louis Althusser ».

\section{JÉRÉMY HAMERS}

J. Hamers est docteur en Arts et sciences de la communication et enseigne l'éducation aux médias ainsi que le cinéma eurégional à l'Université de Liège. La plupart de ses articles concernent le Nouveau Cinéma allemand et la représentation médiatique du terrorisme d'extrême-gauche. Avec Geoffrey Geuens, il a codirigé un dossier sur la radicalité ouvrière, à paraitre dans Quaderni (MSH, 2014).

E-mail: jhamers@ulg.ac.be

Bibliographie complète: http://orbi.ulg.ac.be/ph-search?uid=U196248 